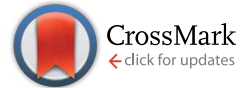

Cite this: RSC Adv., 2017, 7, 13777

Received 24th November 2016 Accepted 13th February 2017

DOI: 10.1039/c6ra27271a

rsc.li/rsc-advances

\section{A first principles study of the interaction between two-dimensional black phosphorus and $\mathrm{Al}_{2} \mathrm{O}_{3}$ dielectric}

\begin{abstract}
Jie Sun, ${ }^{a} \mathrm{Na}$ Lin, ${ }^{* a}$ Cheng Tang, ${ }^{a}$ Hao Ren ${ }^{\mathrm{b}}$ and Xian Zhao*a
The chemical degradation of exfoliated black phosphorus (BP) when exposed to ambient conditions can be effectively suppressed via the deposition of $\mathrm{Al}_{2} \mathrm{O}_{3}$ dielectric on the $\mathrm{BP}$ surface. A good understanding of the interactions between the $\mathrm{BP}$ layer and $\mathrm{Al}_{2} \mathrm{O}_{3}$ dielectric is important for practical device applications. In the presented paper, first principles calculations have been performed to study the structural, energetic, and electronic properties of $\mathrm{BP}$ on an Al-terminated and hydroxylated $\mathrm{Al}_{2} \mathrm{O}_{3}$ (0001) surface. Our calculations indicate the band gap of monolayer BP is enlarged by about $160 \mathrm{meV}$ and $92 \mathrm{meV}$ after the deposition respectively of an Al-terminated and hydroxylated $\mathrm{Al}_{2} \mathrm{O}_{3}$ surface, which is mainly due to interlayer charge transfer between the $\mathrm{BP}$ and $\mathrm{Al}_{2} \mathrm{O}_{3}$ surfaces. However, this trend for increasing band gap is inverted upon increasing the number of $\mathrm{BP}$ layers. Besides, the valence-band offset of a few-layer (2-4 layer) BP/ $\mathrm{Al}_{2} \mathrm{O}_{3}$ system is about $0.5-0.9 \mathrm{eV}$ larger than that of a monolayer $\mathrm{BP} / \mathrm{Al}_{2} \mathrm{O}_{3}$ system, which is more suitable for creating an injection barrier. Moreover, the band gaps of $\mathrm{BP} / \mathrm{Al}_{2} \mathrm{O}_{3}$ systems could be tuned using an external electric field for practical applications.
\end{abstract}

\section{Introduction}

Two-dimensional (2D) black phosphorus (BP) has been studied intensively due to its unique electronic structure and potential for application in nanodevices. ${ }^{1-3}$ In contrast to gapless graphene, BP presents a direct band gap that is capable of manifesting large ON/OFF ratios when being used as a channel material in a field effect transistor (FET). ${ }^{4}$ Meanwhile, its relatively high mobility compared with transition metal dichalcogenides (TMDCS) also makes it an appealing candidate for future devices. ${ }^{5}$ In addition, the layer-sensitive band gap of BP varies with thickness, from $\sim 2 \mathrm{eV}$ for a single layer (termed phosphorene) to $\sim 0.3 \mathrm{eV}$ in bulk form, opening up new opportunities for optoelectronic applications..$^{6-8}$

Despite the praise for BP seeming promising, the nature of defects, ${ }^{9,10}$ contacts $^{11,12}$ and chemical stability ${ }^{13,14}$ in 2D BP are still problematic for realistic device applications. Exfoliated BP flakes have been found to chemically degrade upon exposure to ambient conditions. ${ }^{15,16}$ Therefore, effective protection to prevent the degradation process is critical for 2D BP. Devices, including carbon nanotube and graphene field-effect transistors (FETs), have exhibited improved performance following encapsulation. ${ }^{17-19}$ To date, many efforts toward BP surface

${ }^{a}$ State Key Laboratory of Crystal Materials, Shandong University, 250100 Jinan, Shandong, PR China.E-mail: linnakth@gmail.com; xianzhao@sdu.edu.cn

${ }^{b}$ State Key Laboratory of Heavy Oil Processing \& Center for Bioengineering and Biotechnology, China University of Petroleum (East China), 266580 Qingdao, PR China encapsulation have been reported. For example, recent experiments show that a BP device encapsulated with hexagonal boron nitride (h-BN) possesses high field-effect mobility ( 1350-4000 $\left.\mathrm{cm}^{2}(\mathrm{~V} \mathrm{~s})^{-1}\right)$ and can be stable under an ambient atmosphere for more than 1 week. ${ }^{20}$ In addition, $\mathrm{Al}_{2} \mathrm{O}_{3}$, which has been considered as a suitable dielectric layer for graphene ${ }^{21}$ and TMDCS, ${ }^{22}$ has also been used as a passivation layer in recent $\mathrm{BP}$ transistors. ${ }^{23}$ Luo et al. $^{24}$ and Wood et al. ${ }^{16}$ have mentioned that $\mathrm{Al}_{2} \mathrm{O}_{3}$ passivated few-layer BP FETs can be stable in air for more than 100 hours.

Although it is a promising idea to use $\mathrm{BN}$ or $\mathrm{Al}_{2} \mathrm{O}_{3}$ as a capping layer to protect $\mathrm{BP}$, some fundamental issues, such as interface bonding, charge transfer, band gap change, and band alignment, remain little understood. For device applications based on $\mathrm{BN} / \mathrm{BP}$ and $\mathrm{Al}_{2} \mathrm{O}_{3} / \mathrm{BP}$, understanding these fundamental issues is of high importance and essential. Recent theoretical investigations ${ }^{25}$ have demonstrated that $\mathrm{BN}$ can be used not only as an effective capping layer to protect BP from chemical degradation, maintaining its major electronic characteristics, but also as an active layer to tune the carrier dynamics and optical properties of BP. However, investigations into the physical properties of $\mathrm{Al}_{2} \mathrm{O}_{3} / \mathrm{BP}$ interfaces are still lacking. Recently, Shao et al. ${ }^{26}$ have constructed a bilayer Alterminated $\mathrm{Al}_{2} \mathrm{O}_{3}$ surface model to study the interactions between monolayer $\mathrm{BP}$ and $\mathrm{Al}_{2} \mathrm{O}_{3}$. They found that the electronic properties of $\mathrm{BP}$ were severely changed due to bond formation between the $\mathrm{BP}$ and $\mathrm{Al}_{2} \mathrm{O}_{3}$ surfaces. Nevertheless, $\mathrm{BP}$ on a single layer $\mathrm{Al}$ terminated $\mathrm{Al}_{2} \mathrm{O}_{3}$ surface, which has been demonstrated to be the most stable model of an $\mathrm{Al}_{2} \mathrm{O}_{3}$ (0001) 
surface $^{27}$ and forms no bond with graphene, ${ }^{21}$ is yet to be addressed. Besides, the behavior of $\mathrm{BP}$ on $\mathrm{Al}_{2} \mathrm{O}_{3}$ under a finite electric field is still unclear.

Based on the unexplored issues mentioned above, first principles calculations were carried out in the presented paper to investigate the interactions between $\mathrm{BP}$ and $\mathrm{Al}_{2} \mathrm{O}_{3}$ surfaces. The most stable configurations were determined by calculating the formation energy of $\mathrm{BP} / \mathrm{Al}_{2} \mathrm{O}_{3}$ hybrid systems. The layer distances and charge transfer characteristics between BP and $\mathrm{Al}_{2} \mathrm{O}_{3}$ surfaces were also involved. The electronic properties of $\mathrm{BP}$ affected by the $\mathrm{Al}_{2} \mathrm{O}_{3}$ surface were revealed and band-gap modulations of $\mathrm{BP} / \mathrm{Al}_{2} \mathrm{O}_{3}$ systems under an external electric field were also investigated.

\section{Computational details}

First principles calculations were performed based on density functional theory (DFT) implemented in the Vienna ab initio simulation package (VASP). ${ }^{28}$ The projector-augmented wave (PAW) method was used to describe electron-ion interactions. The generalized gradient approximation in its Perdew, Burke, and Ernzerhof form (GGA-PBE) ${ }^{29}$ was used for the exchangecorrelation part of electron-electron interactions. The van der Waals (vdW) correction proposed by Grimme (DFT-D2) ${ }^{30}$ was chosen to describe long-range interactions. Two relatively stable configurations of an $\mathrm{Al}_{2} \mathrm{O}_{3}$ (0001) surface, namely, a single layer Al-terminated and a fully hydroxylated $\mathrm{Al}_{2} \mathrm{O}_{3}$ (0001) surface (Fig. 1), were considered in our work. The $\mathrm{Al}_{2} \mathrm{O}_{3}$ (0001) surface was modeled using a slab containing six oxygen $\mathrm{O}$ layers and 12 or 11 aluminum layers (depending on the specific surface studied). The second surface of the slab was passivated using pseudo $\mathrm{H}$ atoms. The plane-wave energy cutoff was set to $400 \mathrm{eV}$. A vacuum layer larger than $20 \AA$ was used to avoid interactions between periodic images. Monkhorst-Pack $k$-points of $4 \times 10 \times$ 1 were used for sampling in the first Brillouin zone during geometry optimizations. All geometry structures were fully relaxed until the energy and force were converged respectively to $10^{-6} \mathrm{eV}$ and $0.01 \mathrm{eV} \AA^{-1}$. The dipolar correction has been included.

The formation energy is defined as $E_{\mathrm{f}}=\left(E_{\text {tot }}-E_{\mathrm{BP}}-E_{\mathrm{Al}_{2} \mathrm{O}_{3}}\right) /$ $n$, where $E_{\text {tot }}, E_{\mathrm{BP}}$, and $E_{\mathrm{Al}_{2} \mathrm{O}_{3}}$ are the energies of the $\mathrm{BP} / \mathrm{Al}_{2} \mathrm{O}_{3}$ system, the isolated $\mathrm{BP}$ sheet, and the isolated $\mathrm{Al}_{2} \mathrm{O}_{3}$ substrate, respectively. $n$ is the total number of $\mathrm{P}$ atoms in the system. Therefore, the more negative the formation energy, the more stable the combined system.

\section{Results and discussion}

At the beginning of our work, the selected unit cell lattice constants are calculated at $3.298 \AA \times 4.632 \AA$ for $\mathrm{BP}$ and $8.249 \AA$ $\times 4.762 \AA$ for $\mathrm{Al}_{2} \mathrm{O}_{3}$, respectively. We construct interface models consisting of $5 \mathrm{BP}$ and $2 \mathrm{Al}_{2} \mathrm{O}_{3}$ unit cells with dimensions of $16.49 \AA \times 4.76 \AA$, including a $\sim 2.7 \%$ lattice mismatch. Here the lattice parameter of $\mathrm{Al}_{2} \mathrm{O}_{3}$ is fixed by considering that it is not easy to deform compared with BP. Therefore, the lattice of the BP unit cell along the armchair direction is stretched by $\sim 0.13 \AA$ $(\sim 2.7 \%$ strain $)$, which induces the band gap of $\mathrm{BP}$ in our (a)
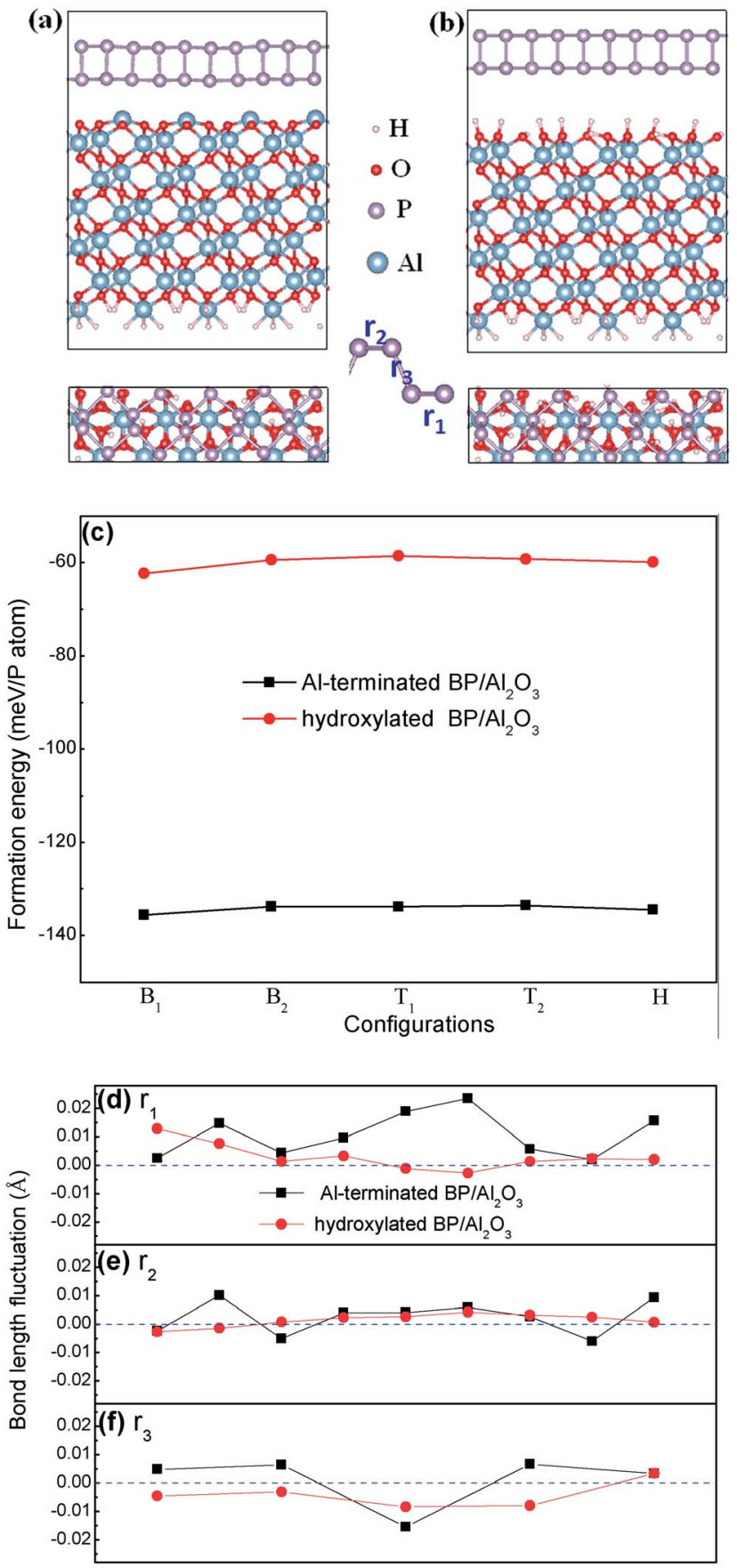

Fig. 1 Side and top views of optimized structures of BP on (a) Alterminated, and (b) fully hydroxylated $\mathrm{Al}_{2} \mathrm{O}_{3}$ (0001) surfaces. (c) The formation energies of $\mathrm{BP}$ adsorbed on different adsorption sites of the Al-terminated and hydroxylated $\mathrm{Al}_{2} \mathrm{O}_{3}$ surfaces. (d)-(f) Bond length alternations of $\mathrm{BP}$ interacting with and without a substrate.

constructed model to enlarge by $\sim 0.1 \mathrm{eV}$ compared with free BP. We think this lattice mismatch is acceptable and using the enlarged band gap as standard will not prevent us from giving reasonable predictions in investigations into substrate and external field effects on the band gap modulation of BP.

After carefully checking the optimized structure of the $\mathrm{Al}_{2} \mathrm{O}_{3}$ (0001) surface, we find that the topmost $\mathrm{Al}$ atoms contract $\sim 81 \%$ after relaxation in the Al-terminated case compared to 
the cleaved surface, as shown in Fig. 1(a), which is consistent with previous studies. ${ }^{31,32}$ The reconstructed surface maintains its stability well due to a net zero surface dipole. ${ }^{33}$ As for the hydroxylated case, the formation of hydroxyls disturbs the order of arrangement of the oxygen atoms. Previous calculations have demonstrated that the stability of a fully hydroxylated surface is comparable to an Al-terminated one. ${ }^{34,35}$ In order to determine the stable interface of $\mathrm{BP} / \mathrm{Al}_{2} \mathrm{O}_{3}$, we place monolayer $\mathrm{BP}$ at five high symmetry positions on top of one $\mathrm{Al}$ or $\mathrm{O}$ atom, that is, two top sites (the two $\mathrm{P}$ atoms $\left(\mathrm{T}_{1 \mathrm{Al}}, \mathrm{T}_{2 \mathrm{Al}}\right.$ or $\left.\mathrm{T}_{1 \mathrm{O}}, \mathrm{T}_{2 \mathrm{O}}\right)$ nearest to the $\mathrm{Al}_{2} \mathrm{O}_{3}$ (0001) surface), two bridge sites (the nearest $\left(\mathrm{B}_{1 \mathrm{Al}}\right.$ or $\left.\mathrm{B}_{1 \mathrm{O}}\right)$ and next-nearest $\left(\mathrm{B}_{2 \mathrm{Al}}\right.$ or $\left.\mathrm{B}_{2 \mathrm{O}}\right) \mathrm{P}-\mathrm{P}$ bond to the $\mathrm{Al}_{2} \mathrm{O}_{3}(0001)$ surface) and one hollow site $\left(\mathrm{H}_{\mathrm{Al}}\right.$ or $\left.\mathrm{H}_{\mathrm{O}}\right)$. Our $\mathrm{PBE}+\mathrm{D} 2$ total energy calculations indicate that the most stable configuration for the Al-terminated surface is $\mathrm{B}_{1 \mathrm{Al}}$ with a formation energy of $-135 \mathrm{meV}$ per $\mathrm{P}$ atom, which is larger than for a graphene/Alterminated $\mathrm{Al}_{2} \mathrm{O}_{3}$ system $(-76.4 \mathrm{meV} \text { per } \mathrm{C} \text { atom })^{21}$ and comparable with a $\mathrm{BP} / \mathrm{Si}$-terminated $\mathrm{SiO}_{2}$ system $(-130 \mathrm{meV}$ per $\mathrm{P}$ atom). ${ }^{36}$ The distance between the BP layer and the substrate surface is $\sim 2.52 \AA$, which is smaller than the typical van der Waals interaction distance $(\sim 3 \AA)$. The formation energy of the $\mathrm{B}_{1 \mathrm{Al}}$ configuration is slightly lower than other configurations by 1-2 meV per $\mathrm{P}$ atom (Fig. 1(c)). The small energy difference indicates that these configurations are almost degenerate in energy. Similar to the Al-terminated surface, the most stable configuration of the fully hydroxylated surface is also the bridge site $\left(B_{10}\right)$. The interlayer distance between the BP layer and the hydroxylated surface ranges from $2.40 \AA$ to $3.26 \AA$, due to the rippled structure at the interface, and the formation energy is $-62.31 \mathrm{meV}$ per $\mathrm{P}$ atom, smaller than the interlayer binding strength in bulk BP ( $~ 81 \mathrm{meV}$ per atom). The formation energy of $\mathrm{B}_{1 \mathrm{O}}$ is more negative than other configurations, by $2-4 \mathrm{meV}$ per $\mathrm{P}$ atom (Fig. 1(c)).

In addition, the bond length alternations of BP interacting with and without a substrate are displayed in Fig. 1(d)-(f). Three types of $\mathrm{P}-\mathrm{P}$ bond, marked as $\mathrm{r}_{1}$ (in the lower BP layer), $\mathrm{r}_{2}$ (in the upper BP layer) and $r_{3}$ (between the two layers) with a number of 9, 9 and 5, are in our constructed model. We note that the P-P bond length alternations are different in the same bond type, due to the fluctuating interface of the substrate. The bond length of $\mathrm{BP}$ is easier to influence when placing it on the Alterminated $\mathrm{Al}_{2} \mathrm{O}_{3}$ surface, compared with on the hydroxylated $\mathrm{Al}_{2} \mathrm{O}_{3}$ surface. The largest bond alternation is found in the lower $\mathrm{BP}$ layer when placing $\mathrm{BP}$ on an Al-terminated $\mathrm{Al}_{2} \mathrm{O}_{3}$ surface, with a value of $\sim 0.028 \AA$. P-P bond fluctuations in the upper BP layer are much softer, indicating weak interactions between the upper BP layer and the substrate. All these bond length alternations contribute to the modulation of the electronic structures of BP.

After obtaining the most stable interface structures, Bader charge analysis ${ }^{37}$ was performed to examine the charge transfer between the $\mathrm{BP}$ and $\mathrm{Al}_{2} \mathrm{O}_{3}$ surfaces. It is found that $\sim 0.138 e$ is transferred from the Al-terminated $\mathrm{Al}_{2} \mathrm{O}_{3}$ surface to $\mathrm{BP}$ and will induce n-type doping of the BP layer. However, for the hydroxylated surface case, the direction of charge transfer is inverted. $\sim 0.092 e$ is transferred from $\mathrm{BP}$ to $\mathrm{Al}_{2} \mathrm{O}_{3}$, leading to p-type doping of the BP layer. The smaller charge transfer in the hydroxylated case compared to the Al-terminated case indicates weaker interaction with BP. To further elucidate the charge transfer between $\mathrm{BP}$ and the $\mathrm{Al}_{2} \mathrm{O}_{3}$ surface, isosurface plots of the electron charge density difference are provided in Fig. 2. The charge density difference $(\Delta \rho)$ is defined as, $\Delta \rho=\rho_{\mathrm{BP} / \mathrm{Al}_{2} \mathrm{O}_{3}}-\rho_{\mathrm{BP}}$ $-\rho_{\mathrm{Al}_{2} \mathrm{O}_{3}}$, where $\rho_{\mathrm{BP} / \mathrm{Al}_{2} \mathrm{O}_{3}}, \rho_{\mathrm{BP}}$ and $\rho_{\mathrm{Al}_{2} \mathrm{O}_{3}}$ denote the charge density of the $\mathrm{BP} / \mathrm{Al}_{2} \mathrm{O}_{3}$ system, the isolated $\mathrm{BP}$ sheet, and the isolated $\mathrm{Al}_{2} \mathrm{O}_{3}$ substrate, respectively. It is observed that charge transfer and redistribution occur mainly between the bottom $\mathrm{P}$ atoms of BP and the top atoms of the substrate surface. Fig. 2(a) shows obvious charge depletion in the four top $\mathrm{Al}$ atoms of the $\mathrm{Al}-$ terminated surface and charge accumulation in the bottom BP layer. As for the hydroxylated case, besides the charge depletion in the bottom BP layer and accumulation around the hydrogen atoms, there is also charge accumulation in the bottom BP layer. This indicates that both intra- and inter-plane charge transfer is occurring in the hydroxylated case. The charge transfer induced by the $\mathrm{Al}_{2} \mathrm{O}_{3}$ surface is expected to have an impact on the electronic properties of BP.

We now turn to discussing the effects of an $\mathrm{Al}_{2} \mathrm{O}_{3}$ substrate on the electronic band structure of BP. The projected band structure of single-layer BP adsorbed on an Al-terminated surface $\left(B_{1 A l}\right.$ configuration) is shown in Fig. 3(a). We notice that the band gap nature of BP transforms from direct to indirect due to a shift in the conduction band minimum from the $\Gamma$ point to the $X$ point, and the band gap value is enlarged by $\sim 160$ meV compared with isolated, free-standing BP (Fig. 3(c)). These band structure changes mainly result from inter-layer charge transfer. The charge-density distributions of the conductionband minimum (CBM) and valence-band maximum (VBM) of $\mathrm{BP}$ on an Al-terminated $\mathrm{Al}_{2} \mathrm{O}_{3}$ surface (Fig. 3(d)) are found to be much more non-uniform compared with that of pure BP (Fig. 3(f)). For the hydroxylated case ( $\mathrm{B}_{10}$ configuration), the band structure nature of $\mathrm{BP}$ is almost unchanged and only a negligible increase in the band gap of $\mathrm{BP}(\sim 84 \mathrm{meV})$ is observed in Fig. 3(b), indicating that hydroxylated $\mathrm{Al}_{2} \mathrm{O}_{3}$ preserves the intrinsic band structure of $\mathrm{BP}$ well, owing to weak
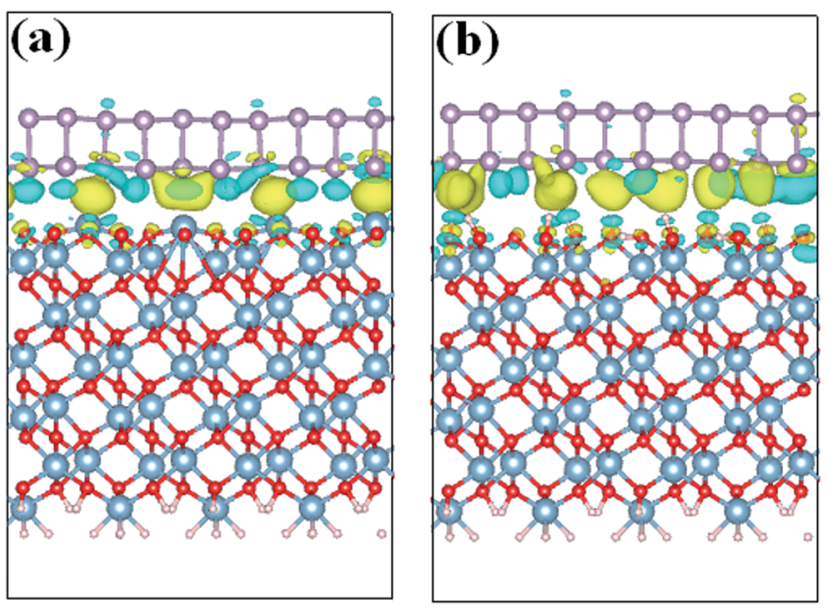

Fig. 2 Charge density differences for $\mathrm{BP}$ adsorbed on (a) Al-terminated, and (b) fully hydroxylated $\mathrm{Al}_{2} \mathrm{O}_{3}$ (0001) surfaces. Blue (yellow) regions show electron accumulation (depletion). 

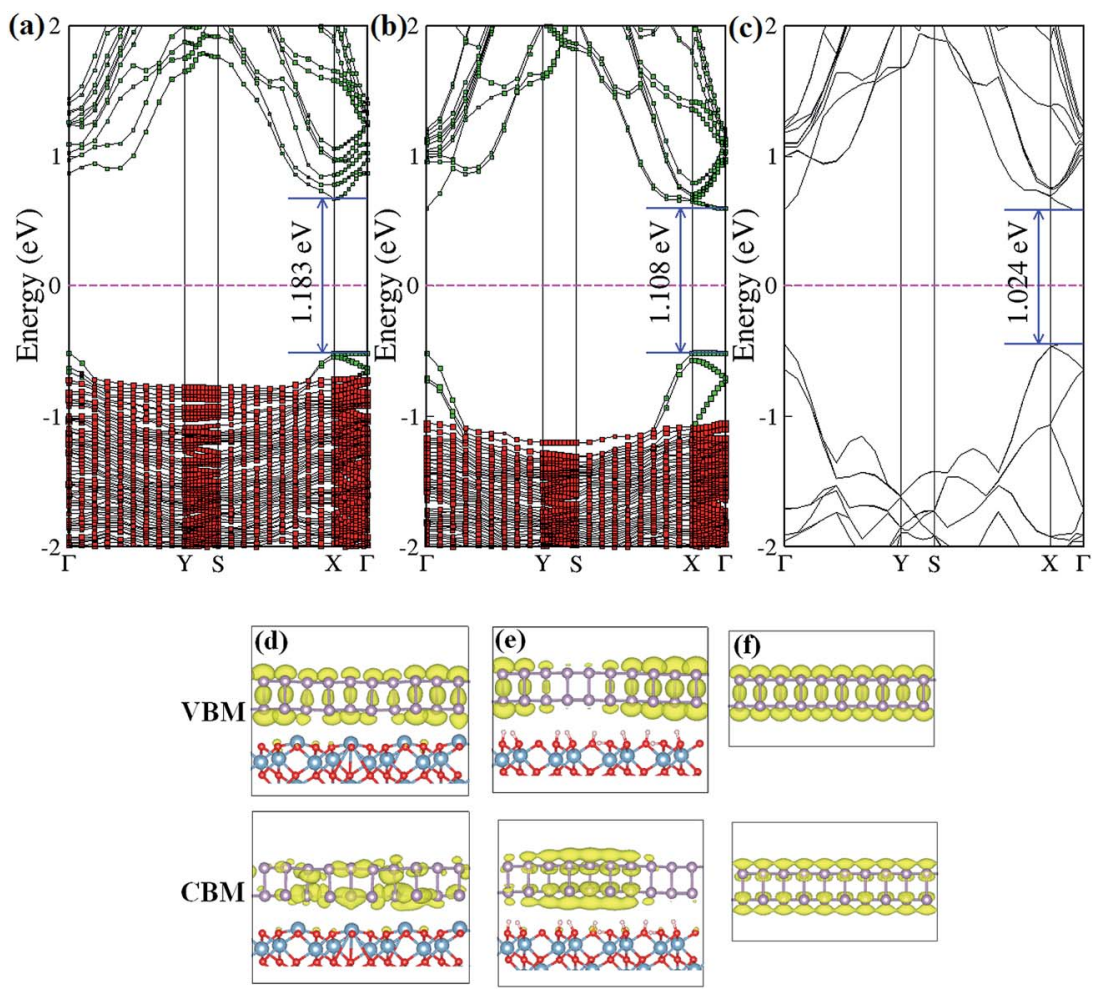

Fig. 3 The band structure of BP adsorbed on (a) Al-terminated, and (b) fully hydroxylated $\mathrm{Al}_{2} \mathrm{O}_{3}$ surfaces, and (c) pure BP band structure. The green squares and red squares represent the band structure projected on $\mathrm{BP}$ and $\mathrm{Al}_{2} \mathrm{O}_{3}$, respectively. (d)-(f) Isosurfaces of the band-decomposed charge density of the valence band maximum and conduction band minimum.

vdW interactions. However, the rippled structure at the interface still makes the corresponding charge-density distributions of the CBM and VBM (Fig. 3(e)) more localized in different parts of the BP layer.

In addition to the most stable configurations, the electronic band natures of other high symmetry configurations of BP on Al-terminated (Fig. 4(a)-(d)) and hydroxylated (Fig. 4(e) and (f)) $\mathrm{Al}_{2} \mathrm{O}_{3}$ surfaces are examined and found to be close to those of $\mathrm{B}_{1 \mathrm{Al}}$ or $\mathrm{B}_{1 \mathrm{O}}$. The differences in the band gap between the most stable configuration and other high symmetry configurations are no larger than $0.01 \mathrm{eV}(0.015 \mathrm{eV})$ for $\mathrm{BP}$ on an Al-terminated (hydroxylated) $\mathrm{Al}_{2} \mathrm{O}_{3}$ surface, indicating that a similar on/off ratio could be obtained even if $\mathrm{BP}$ slides away from its ground state $\mathrm{B}_{1 \mathrm{Al}}\left(\mathrm{B}_{1 \mathrm{O}}\right)$ configuration.

The band gaps of few-layer $\mathrm{BP}$ on Al-terminated and hydroxylated $\mathrm{Al}_{2} \mathrm{O}_{3}$ surfaces are shown in Fig. 5. It can be seen that the band gap of isolated BP decreases with an increasing number of layers, due to enhanced interlayer interactions, which is consistent with previous studies. ${ }^{38}$ We note that this trend does not change when placing few-layer $\mathrm{BP}$ on $\mathrm{Al}$ terminated or hydroxylated $\mathrm{Al}_{2} \mathrm{O}_{3}$ surfaces. Interestingly, the band gaps, which are enlarged by the substrate effect in monolayer BP, gradually reduce upon increasing the number of layers in both the Al-terminated and hydroxylated case, compared with isolated BP layers. For example, the band gaps of monolayer BP on Al-terminated and hydroxylated surfaces are individually increased by $0.16 \mathrm{eV}$ and $0.08 \mathrm{eV}$, while that of fourlayer BP on the two surfaces are decreased by $0.07 \mathrm{eV}$ and
$0.02 \mathrm{eV}$, respectively, compared with isolated BP. After carefully examining the contents of the VBM and CBM of BP, it is found that both the VBM and CBM of free $\mathrm{BP}$ are composed of the $\mathrm{p}_{z}$ orbitals of $\mathrm{P}$ atoms. As $\mathrm{BP}$ is placed on Al-terminated $\mathrm{Al}_{2} \mathrm{O}_{3}$, the $\mathrm{p}_{z}$ orbitals in the VBM are hardly affected by interlayer interactions, but those in the CBM are suppressed, and its energy is largely increased, leading to a new CBM arising and the band gap of BP enlarging. However, for few-layer BP, the interlayer interactions have little influence on the CBM and the decreased band gaps are mainly due to interlayer charge transfer. Furthermore, the band gap reduction for few-layer $\mathrm{BP}$ on an $\mathrm{Al}-$ terminated $\mathrm{Al}_{2} \mathrm{O}_{3}$ surface is larger than on the hydroxylated $\mathrm{Al}_{2} \mathrm{O}_{3}$ surface, due to stronger interactions between the BP and Al-terminated $\mathrm{Al}_{2} \mathrm{O}_{3}$ surface. Moreover, it is worth mentioning that the band gap of bilayer BP is nearly independent of the substrate, indicating that it may be a potential candidate for special device applications.

In comparison with band structures, the band offset of semiconducting heterostructures is also very important in material and device design. ${ }^{39}$ Precise knowledge is extremely important to engineer electronic and optoelectronic devices. The band alignments of a $\mathrm{BP} / \mathrm{Al}_{2} \mathrm{O}_{3}$ system have been calculated using the macroscopic averaging method. ${ }^{40}$ The electrostatic potential has been chosen as a reference, the change in the average electrostatic potential through the interface is obtained through calculating the $\mathrm{BP} / \mathrm{Al}_{2} \mathrm{O}_{3}$ heterostructures, and the VBMs of the two semiconductors with respect to the electrostatic potential have been calculated using their individual 

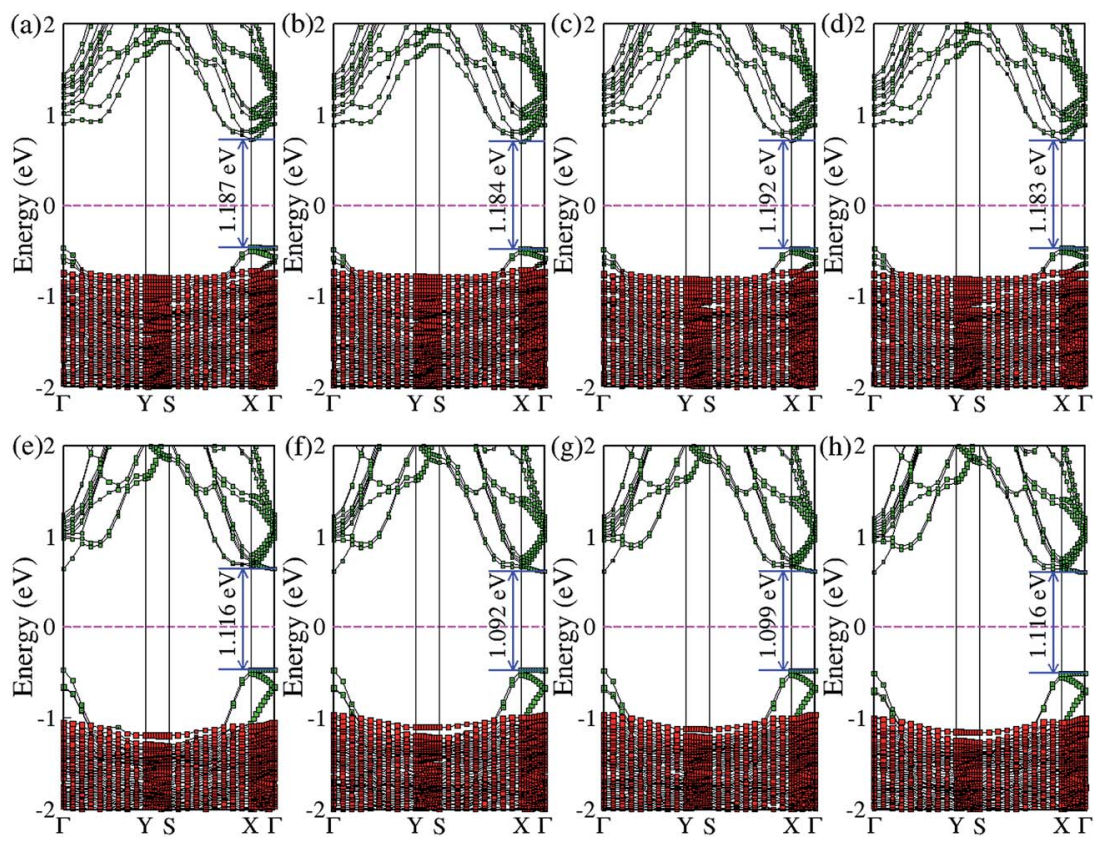

Fig. 4 The band structures of other high symmetry configurations of BP on Al-terminated (a)-(d) and hydroxylated (e)-(h) $\mathrm{Al}_{2} \mathrm{O}_{3}$ surfaces. The green and red squares represent the band structure projected on $\mathrm{BP}$ and $\mathrm{Al}_{2} \mathrm{O}_{3}$, respectively.

supercells. The calculated results in Fig. 6 show that the macroscopic average potential is discontinuous at the interface of $\mathrm{BP}$ and $\mathrm{Al}_{2} \mathrm{O}_{3}$, indicating that electrons should overcome a potential barrier (3.5-3.8 eV) to cross from $\mathrm{Al}_{2} \mathrm{O}_{3}$ to the $\mathrm{BP}$ section. We note that the VBM of $\mathrm{BP}$ is higher than that of $\mathrm{Al}_{2} \mathrm{O}_{3}$, which indicates that $\mathrm{BP}$ and Al-terminated $\mathrm{Al}_{2} \mathrm{O}_{3}$ form a type I heterointerface, due to a large band gap in bulk $\mathrm{Al}_{2} \mathrm{O}_{3}$ (our DFT $+\mathrm{PBE}$ result is $6.2 \mathrm{eV}$ ). The predicted valence-band offset (VBO) $\Delta E_{\mathrm{V}}$ between monolayer $\mathrm{BP}$ and $\mathrm{Al}_{2} \mathrm{O}_{3}$ is about $0.4 \mathrm{eV}$ (Fig. 6(a)), which is smaller than that of graphene $/ \mathrm{Al}_{2} \mathrm{O}_{3}$ and $\mathrm{MoS}_{2} / \mathrm{Al}_{2} \mathrm{O}_{3}$. This value may not be ideal for practical FET devices based on BP material. For practical use, the valence band offset is expected to be sufficiently large $(>1.0 \mathrm{eV})$, which allows suppression of electron thermal emission from channel to the gate or reduction of the gate leakage current. As the number of $\mathrm{BP}$ layers increases, the VBO of few-layer BP is enlarged, but not monotonic. The VBO values are 1.32, 0.96 and $1.15 \mathrm{eV}$ for bilayer, three-layer and four-layer BP, respectively (Fig. 6(b)-(d)), which is more appropriate for creating a reasonable carrier injection barrier.

Finally, we considered the electronic properties of a $\mathrm{BP} / \mathrm{Al}_{2} \mathrm{O}_{3}$ hybrid system under an external electric field $\left(E_{\text {ext }}\right)$ to simulate the gating effect in experiments. Fig. 7 shows the band gaps of $\mathrm{BP} / \mathrm{Al}_{2} \mathrm{O}_{3}$ systems as a function of $E_{\text {ext }}$. The positive (negative) values of $E_{\text {ext }}$ represent the direction of $E_{\text {ext }}$ vertical to the BP plane and pointing from $\mathrm{Al}_{2} \mathrm{O}_{3}(\mathrm{BP})$ to $\mathrm{BP}\left(\mathrm{Al}_{2} \mathrm{O}_{3}\right)$. For monolayer $\mathrm{BP}$ on an Al-terminated $\mathrm{Al}_{2} \mathrm{O}_{3}$ surface, the band gaps show negligible changes under a negative $E_{\text {ext }}$; this trend is destroyed under a positive $E_{\text {ext }}$, as $E_{\text {ext }}>0.3 \mathrm{~V} \mathrm{\AA}^{-1}$, which decreases the band gap. The band gap variations for monolayer $\mathrm{BP}$ on a hydroxylated $\mathrm{Al}_{2} \mathrm{O}_{3}$ surface undergo a similar trend as for the Al-terminated case. However, they seem more sensitive to $E_{\text {ext }}$, with a larger reduction in band gap found as $E_{\text {ext }}>0.3 \mathrm{~V}^{-1}$. For example, the band gap in the hydroxylated case decreases by about $0.34 \mathrm{eV}$ under $E_{\text {ext }}=0.6 \mathrm{~V} \AA^{-1}$, while that value for the Alterminated case is only $0.14 \mathrm{eV}$ under the same $E_{\text {ext }}$. This is mainly because the distance between the monolayer BP and hydroxylated $\mathrm{Al}_{2} \mathrm{O}_{3}$ surface is more severely decreased compared to that for an Al-terminated surface under $E_{\text {ext }}=0.6 \mathrm{~V}$ $\AA^{-1}$, due to the relatively weak vdW interactions. We have checked that the reduction distances are $0.34 \AA$ and $0.14 \AA$ for the hydroxylated and Al-terminated case under $E_{\text {ext }}=0.6 \mathrm{~V}^{-1}$, respectively. The changed interlayer distance will cause interlayer charge transfer and redistribution, thus changing the

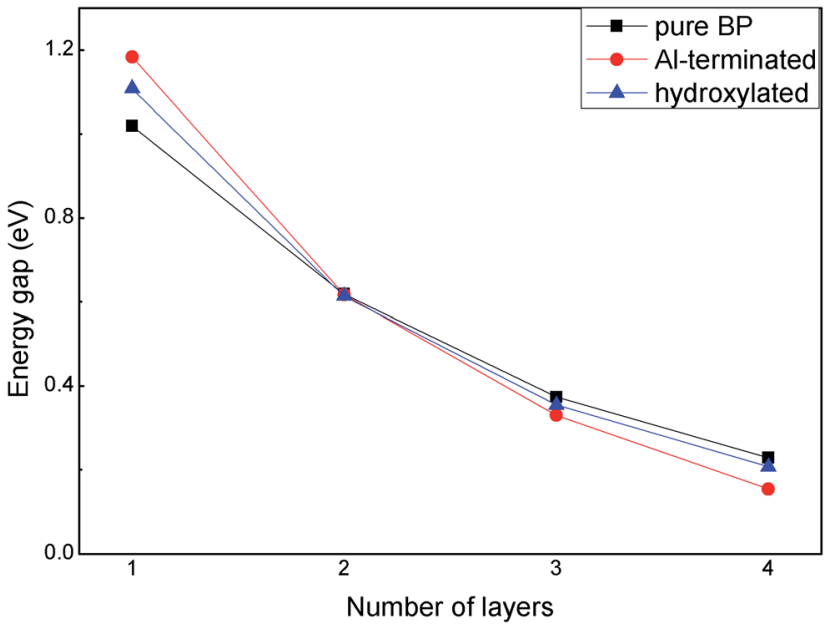

Fig. 5 The band gaps of few-layer BP adsorbed on Al-terminated and hydroxylated $\mathrm{Al}_{2} \mathrm{O}_{3}$ surfaces. 

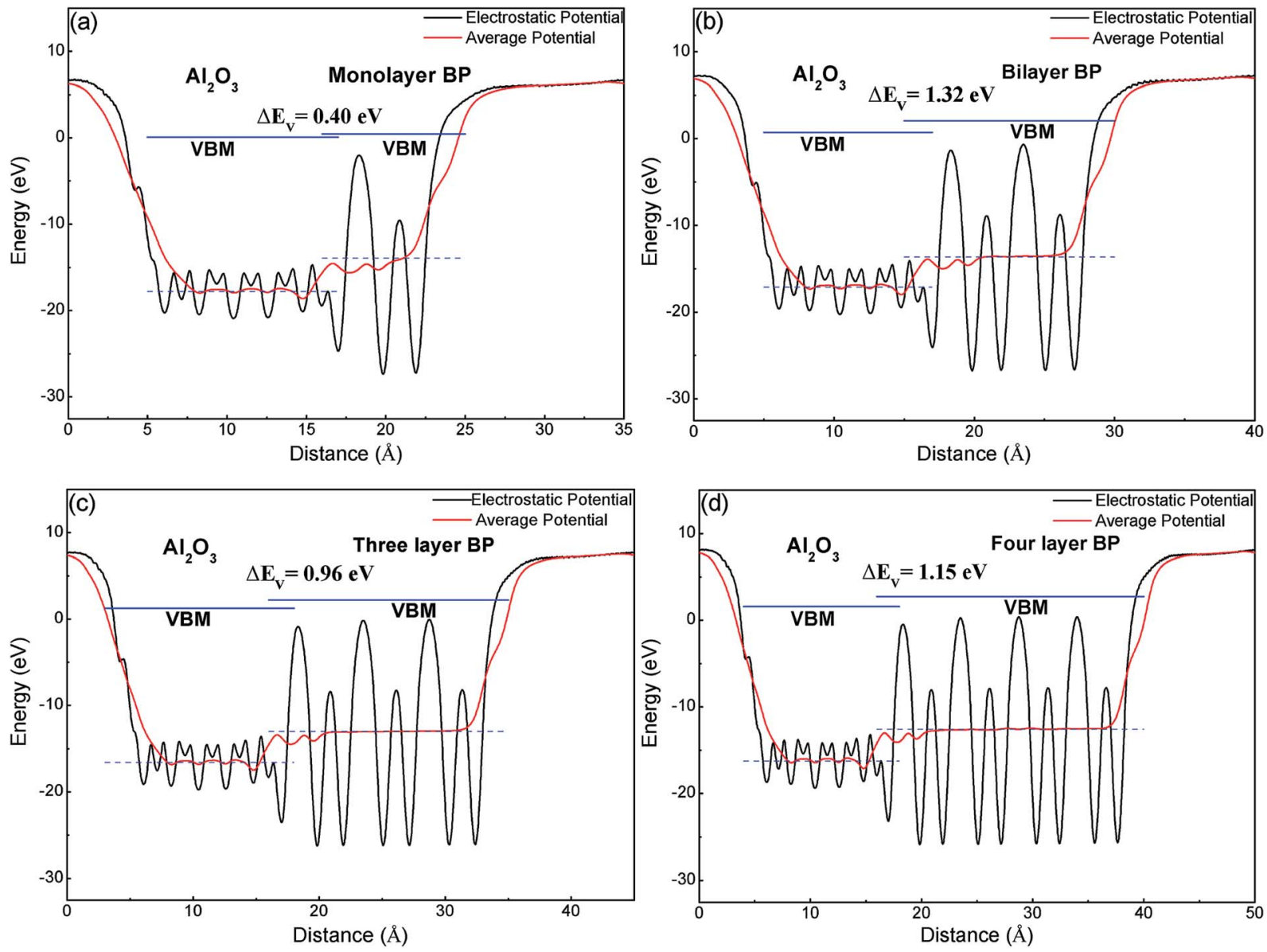

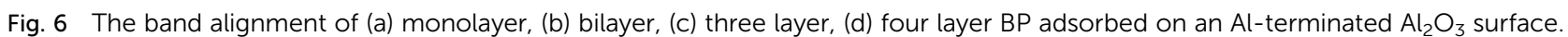

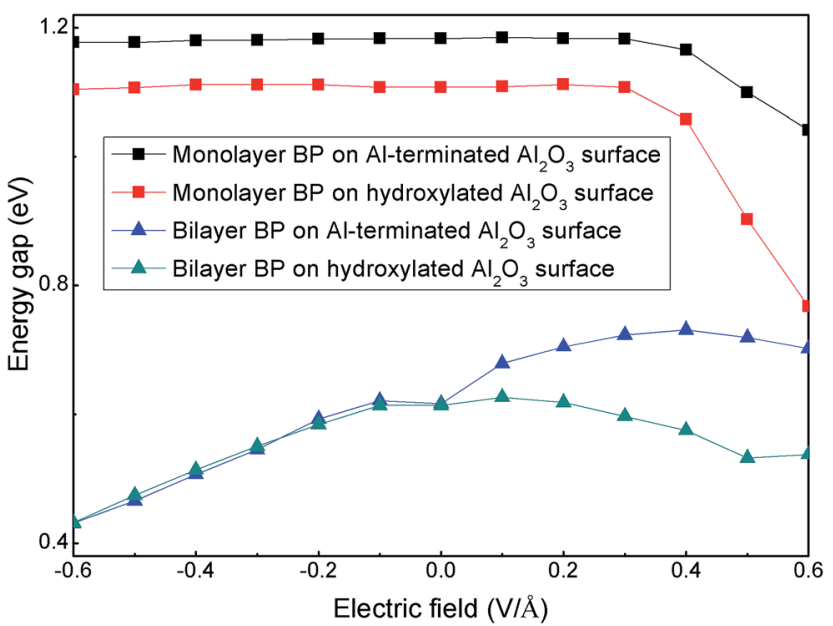

Fig. 7 The band gaps of monolayer and bilayer BP adsorbed on Alterminated and hydroxylated $\mathrm{Al}_{2} \mathrm{O}_{3}$ surfaces as a function of external electric field.

band gap. The situation is quite different for bilayer $\mathrm{BP} / \mathrm{Al}_{2} \mathrm{O}_{3}$, in which case we note that the band gaps of $\mathrm{BP}$ on Al-terminated and hydroxylated $\mathrm{Al}_{2} \mathrm{O}_{3}$ surfaces under a negative $E_{\text {ext }}$ are nearly the same, both monotonically decreasing upon an increasing
$E_{\text {ext }}$ value. Under a positive $E_{\text {ext }}$, the band-gap variations for BP on an Al-terminated $\mathrm{Al}_{2} \mathrm{O}_{3}$ surface show an increasing and then decreasing trend. For BP on a hydroxylated $\mathrm{Al}_{2} \mathrm{O}_{3}$ surface, the band-gap modulations are analogous to those under a negative $E_{\text {ext }}$, decreasing but with a more smooth trend. Therefore, the electronic properties of BP rely on the substrate interface, as modulated by the external electric field. Though the nonbonding model of the $\mathrm{BP} / \mathrm{Al}_{2} \mathrm{O}_{3}$ hybrid system in our work is not precise enough for a thorough description of the interactions between $\mathrm{BP}$ and the $\mathrm{Al}_{2} \mathrm{O}_{3}$ surface under practical experiment, which may include defects, adsorbates, oxides in the interface and the formation of bond states, it still gives useful information on how the electronic properties of the BP layer are affected by an $\mathrm{Al}_{2} \mathrm{O}_{3}$ dielectric, which we think may contribute to practical device design.

\section{Conclusions}

In summary, first principles calculations have been performed to systematically study the electronic properties of $\mathrm{BP}$ on $\mathrm{Al}-$ terminated and hydroxylated $\mathrm{Al}_{2} \mathrm{O}_{3}$ surfaces. We found that the Al-terminated $\mathrm{Al}_{2} \mathrm{O}_{3}$ surface would induce a shift of the conduction band minimum of $\mathrm{BP}$, while a hydroxylated $\mathrm{Al}_{2} \mathrm{O}_{3}$ surface could maintain well the intrinsic band nature of BP. The 
band gaps of $\mathrm{BP}$ on the two surfaces are both enlarged, due to interlayer charge transfer between $\mathrm{BP}$ and $\mathrm{Al}_{2} \mathrm{O}_{3}$. However, the band gaps of few-layer BP (2-4 layers) were decreased compared with isolated BP in both the Al-terminated and hydroxylated case. The band offset of a monolayer $\mathrm{BP} / \mathrm{Al}_{2} \mathrm{O}_{3}$ system was predicted to be $0.4 \mathrm{eV}$, which was probably not ideal for practical FET devices, however, increased band offsets were found in fewlayer BP, making it more suitable for creating an injection barrier. Moreover, the band gaps of a $\mathrm{BP} / \mathrm{Al}_{2} \mathrm{O}_{3}$ system could be modulated using an external electric field for practical applications.

\section{Acknowledgements}

We acknowledge the National Nature Science Foundation of China (Grant No. 21573129 and 21403300), the National Nature Science Foundation of Shandong Province (Grant No. ZR2015BQ001), and the General Financial Grant from the China Postdoctoral Science Foundation (Grant No. 2013M531595). The authors also acknowledge a generous grant of computer time from the National Supercomputer Center in TianjinTianHe-1(A) and the Norwegian Programme for Supercomputing.

\section{References}

1 L. K. Li, Y. J. Yu, G. J. Ye, Q. Q. Ge, X. D. Ou, H. Wu, D. L. Feng, X. H. Chen and Y. B. Zhang, Nat. Nanotechnol., 2014, 9, 372-377.

2 Y. C. Du, H. Liu, Y. X. Deng and P. D. Ye, ACS Nano, 2014, 8, 10035-10042.

3 H. Liu, A. T. Neal, Z. Zhu, Z. Luo, X. F. Xu, D. Tománek and P. D. Ye, ACS Nano, 2014, 8, 4033-4041.

4 K. S. Novoselov, A. K. Geim, S. V. Morozov, D. Jiang, Y. Zhang, S. V. Dubonos, I. V. Grigorieva and A. A. Firsov, Science, 2004, 306, 666-669.

5 B. Radisavljevic, A. Radenovic, J. Brivio, V. Giacometti and A. Kis, Nat. Nanotechnol., 2011, 6, 147-150.

6 M. Buscema, D. J. Groenendijk, S. I. Blanter, G. A. Steele, H. S. J. van der Zant and A. Castellanos-Gomez, Nano Lett., 2014, 14, 3347-3352.

7 S. Zhang, J. Yang, R. J. Xu, F. Wang, W. F. Li, M. Ghufran, Y. W. Zhang, Z. F. Yu, G. Zhang, Q. H. Qin and Y. R. Lu, ACS Nano, 2014, 8, 9590-9596.

8 V. Tran, R. Soklaski, Y. F. Liang and L. Yang, Phys. Rev. B: Condens. Matter Mater. Phys., 2014, 89, 235319.

9 S. J. Yuan, A. N. Rudenko and M. I. Katsnelson, Phys. Rev. B: Condens. Matter Mater. Phys., 2015, 91, 115436.

10 V. Wang, Y. Kawazoe and W. T. Geng, Phys. Rev. B: Condens. Matter Mater. Phys., 2015, 91, 045433.

11 D. Xiang, C. Han, J. Wu, S. Zhong, Y. Y. Liu, J. D. Lin, X. A. Zhang, W. P. Hu, B. Özyilmaz, A. H. Castro Neto, A. T. S. Wee and W. Chen, Nat. Commun., 2015, 6, 6458.

12 D. J. Perello, S. H. chae, S. Song and Y. H. Lee, Nat. Commun., 2015, 6, 7809.

13 J. O. Island, G. A. Steele, H. S. J. van der Zant and A. Castellanos-Gomez, 2D Mater., 2015, 2, 011002.
14 P. Li, D. Z. Zhang, J. J. Liu, H. Y. Chang, Y. E. Sun and N. L. Yin, ACS Appl. Mater. Interfaces, 2015, 7, 24396-24402. 15 S. P. Koenig, R. A. Doganov, H. Schmidt, A. H. Castro Neto and B. Özyilmaz, Appl. Phys. Lett., 2014, 104, 103106.

16 J. D. Wood, S. A. Wells, D. Jariwala, K. S. Chen, E. K. Cho, V. K. Sangwan, X. L. Liu, L. J. Lauhon, T. J. Marks and M. C. Hersam, Nano Lett., 2014, 14, 6964-6970.

17 A. Javey, H. Kim, M. Brink, Q. Wang, A. Ural, J. Guo, P. McIntyre, P. McEuen, M. Lundstrom and H. J. Dai, Nat. Mater., 2002, 1, 241-246.

18 V. K. Sangwan, D. Jariwala, K. Everaerts, J. J. McMorrow, J. T. He, M. Grayson, L. J. aluhon, T. J. Marks and M. C. Hersam, Appl. Phys. Lett., 2014, 104, 083503.

19 S. Kim, J. Nah, I. Jo, D. Shahrjerdi, L. Colombo, Z. Yao, E. Tutuc and S. K. Banerjee, Appl. Phys. Lett., 2009, 94, 062107. 20 R. A. Doganov, S. P. Koenig, Y. Yeo, K. Watanabe, T. Taniguchi and B. Özyilmaz, Appl. Phys. Lett., 2015, 106, 083505.

21 B. Huang, Q. Xu and S. H. Wei, Phys. Rev. B: Condens. Matter Mater. Phys., 2011, 84, 155406.

22 A. K. Singh, R. G. Henig, A. V. Davydov and F. Tavazza, Appl. Phys. Lett., 2015, 107, 053106.

23 H. Liu, A. T. Neal, M. W. Si, Y. C. Du and P. D. Ye, IEEE Electron Device Lett., 2014, 35, 795-797.

24 X. Luo, Y. Rahbarihagh, J. C. M. Hwang, H. Liu, Y. C. Du and P. D. Ye, IEEE Electron Device Lett., 2014, 35, 1314-1316.

25 Y. Q. Cai, G. Zhang and Y. W. Zhang, J. Phys. Chem. C, 2015, 119, 13929-13936.

26 L. Shao, H. G. Ye, Y. L. Wu, Y. X. Du, P. Ding, F. G. Zeng and Q. X. Yuan, Mater. Res. Express, 2016, 3, 025013.

27 P. J. Eng, T. P. Trainor, G. E. Brown Jr, G. A. Waychunas, M. Newville, S. R. Sutton and M. L. Rivers, Science, 2000, 288, 1029-1033.

28 G. Kresse and J. Hafner, Phys. Rev. B: Condens. Matter Mater. Phys., 1993, 47, 558-561.

29 J. P. Perdew, K. Bruke and M. Ernzerhof, Phys. Rev. Lett., 1996, 77, 3865-3868.

30 S. Grimme, Comput. Chem., 2006, 27, 1787-1799.

31 J. Ahn and J. W. Rabalais, Surf. Sci., 1997, 388, 121-131.

32 P. Guénard, G. Renaud, A. Barbier and M. Gautier-Soye, Surf. Rev. Lett., 1998, 05, 321.

33 M. Gautier, G. Fenaud, L. P. Van, B. Villette, M. Pollak, N. Thromat, F. Jollet and J. P. Duraud, J. Am. Ceram. Soc., 1994, 77, 323-334.

34 R. D. Felice and J. E. Northrup, Phys. Rev. B: Condens. Matter Mater. Phys., 1999, 60, 16287.

35 X. G. Wang, A. Chaka and M. Scheffler, Phys. Rev. Lett., 2000, 84, 3650-3653.

36 Y. J. Kang, J. Kang and K. J. Chang, Phys. Rev. B: Condens. Matter Mater. Phys., 2008, 78, 115404.

37 E. Sanville, S. D. Kenny, R. Smith and G. Henkelman, J. Comput. Chem., 2007, 28, 899-908.

38 J. S. Qiao, X. H. Kong, Z. X. Hu, F. Yang and W. Ji, Nat. Commun., 2014, 5, 4475.

39 A. Franciosi and C. G. Van de Walle, Surf. Sci. Rep., 1996, 25, 1-140.

40 A. Baldereschi, S. Baroni and R. Resta, Phys. Rev. Lett., 1988, 61, 734-737. 